\title{
Avaliação da influência dos estímulos sensoriais envolvidos na manutenção do equilíbrio corporal em mulheres idosas
}

Evaluation the influence sensory stimuli that keep body balance in elderly women

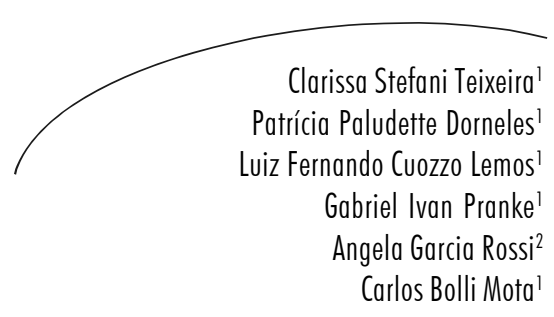

Resumo

Com o envelhecimento, algumas habilidades são comprometidas, como as áreas que processam os sinais dos sistemas vestibular, visual e proprioceptivo. $\mathrm{O}$ objetivo deste estudo foi avaliar o equilíbrio corporal de idosas durante a manipulação dos sistemas sensoriais. Participaram do estudo 39 idosas praticantes de hidroginástica. Uma plataforma de força AMTI foi utilizada para avaliar as variáveis referentes ao equilíbrio corporal na direção ânteroposterior e médio-lateral. O conflito sensorial foi realizado em seis testes de organização sensorial (TOS) por meio da posturografia dinâmica validada por Castagno (1994). Os resultados mostraram que, na direção ântero-posterior, não houve diferença estatisticamente significativa apenas nas comparações do TOS 2 com o TOS 1 , TOS 3 e TOS 4; TOS 3 com o TOS 4 ; e TOS 5 com o TOS 6. Com relação à direção médio-lateral, não foram encontradas diferenças estatisticamente significativas comparando o TOS 1 com o TOS 2 e 3; o TOS 2 comparado ao TOS 3; e quando o TOS 5 foi comparado com o TOS 4 e 6. Durante o TOS, seis idosas apresentaram maior média da amplitude do deslocamento do COP em ambas as direções, o que indica maior instabilidade na manipulação das aferências proprioceptivas e vestibulares. Concluiu-se que quanto mais sistemas sensoriais forem manipulados ou suprimidos, pior será o equilíbrio corporal das idosas.

\footnotetext{
Centro de Educação Física e Desportos. Departamento de Métodos e Técnicas Desportivas. Universidade Federal de Santa Maria. Santa Maria, RS, Brasil

2 Centro de Ciências da Saúde, Departamento de Otorrino-Fonoaudiologia. Universidade Federal de Santa Maria. Santa Maria, RS, Brasil

Palavras-chave: Cinética. Atividade Motora. Saúde do Idoso. Agnosia. Idosas. Equilíbrio músculo esquelético. 


\section{Abstract}

With the aging some skills are compromised, such as areas that process the signs of the vestibular, vision and proprioceptive systems. This study aimed to to evaluate the body balance of elderly women during manipulation of sensorial systems. The study included 39 elderly women who practice hydro-gymnastics. $\mathrm{Na}$ AMTI force platform was used to evaluate the variables related to body balance in the antero-posterior and medium-lateral sense. The sensory conflict was accomplished through six sensorial organization tests (SOT) through the dynamic posturography validated by Castagno (1994). Results showed that in the antero-posterior direction there was no statistically significant differences only in the comparisons of SOT 2 with SOT 1, SOT 3 and SOT 4; in SOT 3 with SOT 4 ; and in SOT 5 with SOT 6 . With respect to the medium-lateral direction there were no statistically significant differences just in the comparison of SOT 1 with SOT 2 and 3; in SOT 2 with SOT 3; and in SOT 5 with SOT 4 and 6. During TOS, six elderly showed higher average amplitude of COP displacement width in both directions, indicating greater instability in the manipulation of proprioceptive and vestibular inputs. We concluded that the more sensory systems are manipulated or suppressed, the worse will be the elderly's body balance.
Key words: Kinetics. Motor Activity. Health of the Elderly. Agnosia. Elderly. Musculoskeletal equilibrium.
INTRODUÇÃOO

A expectativa de vida vem crescendo sistematicamente, gerando aumento percentual na população idosa. Juntamente com a mudança populacional e ao aumento de indivíduos com mais de 60 anos, busca-se uma melhora na qualidade de vida, para a manutenção de um estilo de vida mais independente e saudável. ${ }^{1}$

O envelhecimento compromete determinadas habilidades do sistema nervoso central (SNC), como as áreas que realizam os processamentos de sinais dos sistemas vestibular, visual e proprioceptivo. Esses sistemas corporais são responsáveis pela manutenção do equilíbrio corporal e, quando afetados, agem de forma negativa na capacidade de modificações dos reflexos adaptativos. Devido à ocorrência dos processos degenerativos, o idoso pode sofrer de vertigem e/ou tontura e de desequilíbrios corporais, ${ }^{2}$ que podem causar quedas, fraturas, $3,4,5$ perda de mobilidade, imobilizações e dependência de terceiros para a realização de atividades cotidianas. ${ }^{6,7}$

Diversas alterações nos sistemas motores e sensoriais ocorrem devido ao processo de envelhecimento, mas é difícil descrever quais mudanças são as principais responsáveis pela diminuição da estabilidade postural dessa população. Alguns autores ${ }^{8-11}$ sugerem que esses déficits podem estar relacionados às alterações estruturais e funcionais nos sistemas sensoriais e motor, além de problemas na integração das informações sensoriais. No entanto, mesmo havendo uma rica literatura em torno da estabilidade em pessoas idosas, as principais causas do desequilíbrio não são totalmente conhecidas. ${ }^{9}$ Diante disso, este estudo objetivou avaliar o equilíbrio corporal de mulheres idosas durante a manipulação dos sistemas sensoriais.

\section{METODOLOGIA}

Os indivíduos participantes de grupos de exercícios físicos de uma instituição federal de ensino superior da Região Sul do Brasil foram convidados a participar do estudo. A hidroginástica foi a prática física realizada pelos idosos com uma frequência de duas vezes na semana. Para o controle do nível de atividade física, foi utilizado o Questionário Internacional de Atividades Físicas (IPAQ) e, além disso, como recomendam Benedetti, Mazo e Barros, ${ }^{12}$ por se tratar de uma amostra pequena, além da aplicação 
formal do IPAQ, foram realizadas perguntas individuais em forma de entrevista que permitissem visualizar mais amplamente as atividades físicas diárias dos indivíduos.

De um total de 56 indivíduos de ambos os gêneros, foram avaliados aqueles que aceitaram em participar do estudo e que se propuseram a comparecer nas avaliações. Para os 51 indivíduos que aceitaram a participação voluntária do estudo, foram marcados dia e horário em ambiente de laboratório. Os indivíduos assinaram o Termo de Consentimento Livre e Esclarecido (aprovado sob protocolo 0044.0243.000-07, no Comitê de Ética e Pesquisa de uma instituição federal de ensino superior) e preencheram uma ficha de dados pessoais, para a obtenção do nome, gênero, estatura e idade.
Todos os indivíduos passaram por avaliação otorrinolaringológica. Por meio de uma ficha de anamnese, foram excluídos do estudo dois indivíduos com problemas relacionados a aneurisma, dois com isquemia transitória, um com problema músculoesquelético na região dos membros inferiores e cinco indivíduos que faziam uso de medicamentos associados ao aparelho vestibular.

Além disso, para a exclusão de síndromes vestibulares os indivíduos foram conduzidos a avaliações fonoaudiológicas, para realização de exames de vectoelectonistagmografia, sendo que dois indivíduos foram excluídos por alteração. Diante dessas avaliações, participaram do grupo de estudo 39 mulheres idosas. A idade, a estatura corporal e a massa corporal das idosas estão ilustradas descritas na tabela 1 :

Tabela 1 - Média e desvio padrão da idade, estatura corporal e massa corporal das 39 idosas avaliadas. Santa Maria, RS, 2009.

\begin{tabular}{cccc}
\hline & Idade (anos) & Estatura corporal $(\mathrm{m})$ & Massa corporal $(\mathrm{kg})$ \\
\hline Média & 68,03 & 1,54 & 70,49 \\
Desvio Padrão & 6,39 & 0,07 & 10,60 \\
\hline
\end{tabular}

A mensuração das variáveis referentes ao equilíbrio corporal foi realizada utilizando-se uma plataforma de força OR6-5 AMTI (Advanced Mechanical Technologies, Inc). O conflito sensorial foi realizado por meio do teste de organização sensorial (TOS), utilizando a posturografia dinâmica validada por Castagno. ${ }^{13}$ Para a avaliação, o indivíduo é posicionado sob a plataforma de força dentro de uma cabine de 1 $\mathrm{m}^{2}$, com altura de $2 \mathrm{~m}$, confeccionada com suporte de ferro desmontável, envolta por um tecido de algodão com listras horizontais, claras e escuras de $10 \mathrm{~cm}$ cada uma. A cabine é um sistema mecânico simples e move-se $20^{\circ}$ manualmente para frente e para trás durante a realização dos TOS 3 e 6. Para realizar a manipulação do sistema somatossensorial nos TOS 4, 5 e 6 , foi utilizada uma almofada de $10 \mathrm{~cm}$ de espessura, de $50 \mathrm{~cm} \mathrm{x}$ $50 \mathrm{~cm}$, entre os pés do indivíduo e a plataforma de força. Além disso, no TOS 2 e 4, é solicitado aos indivíduos a permanência sobre o instrumento com ausência de visão, ou seja, com os olhos fechados.

Todas as seis condições testadas, realizadas na ordem de 1 a 6 , são realizadas em posição ortostática com olhos fixos na horizontal. Para a coleta de dados, todos os indivíduos se mantiveram com os pés descalços separados na largura do quadril. Além disso, a plataforma de força foi marcada na primeira tentativa de cada indivíduo para que o posicionamento dos membros inferiores fosse repetido em todas as condições. O tempo de aquisição dos dados para cada tentativa foi de 20 segundos, após a estabilização visual do centro de pressão (COP), a uma frequência de aquisição de $100 \mathrm{~Hz}$. 
Os dados foram utilizados no cálculo de duas coordenadas do centro de pressão a cada instante, uma na direção ântero-posterior e outra na direção médio-lateral, de acordo com o sistema de coordenadas que o software da própria plataforma fornece. A partir desses dados, podem-se obter informações sobre o equilíbrio do indivíduo. A amplitude do deslocamento do COP resultou em duas variáveis utilizadas: amplitude de deslocamento do COP na direção ânteroposterior (COPap) e amplitude de deslocamento do COP na direção médio-lateral (COPml). O cálculo do COP a cada instante é dado pela fórmula:

$$
\begin{gathered}
\text { COPap }=\frac{(M x-(Z o f f * F y))}{F z} \\
\text { COPml }=\left[\left(M y+\left(\text { Zoff }^{*} F x\right)\right) / F z\right] *(-1)
\end{gathered}
$$

Onde:

COPap $=$ coordenada do centro de pressão na direção ântero-posterior;

$C O P m l=$ coordenada do centro de pressão na direção médio-lateral;

Zoff $=$ a distância vertical entre o topo $\mathrm{da}$ plataforma e sua origem (valor negativo);

$$
\begin{aligned}
& F x=\text { a força ao longo do eixo } \mathrm{x} \\
& F_{y}=\text { a força ao longo do eixo } \mathrm{y} ; \\
& F_{z}=\text { a força ao longo do eixo } \mathrm{z} ; \\
& M x=\text { o momento sobre o eixo } \mathrm{x} ; \\
& M_{y}=\text { o momento sobre o eixo } \mathrm{y} ; \\
& M_{z}=\text { o momento sobre o eixo } \mathrm{z} ;
\end{aligned}
$$

Para análise dos dados, foi utilizada a estatística descritiva. A normalidade dos dados foi verificada por meio do teste de KolmogorovSmirnov. Como os dados não apresentaram distribuição normal, foi utilizado o teste ANOVA de Friedman para comparação das variáveis do estudo. O nível de significância utilizado para todos os testes foi de $5 \%$.

\section{RESULTADOS}

A descrição das variáveis relacionadas ao equilíbrio corporal, nas seis condições sensoriais, está disposta na tabela 2. Além disso, na mesma tabela estão apresentadas as comparações realizadas entre os seis TOS, para a COPap e COPml, cujas letras sobrescritas, quando diferentes, indicam diferenças estatisticamente significativas entre os TOS. 
Tabela 2 - Média e desvio padrão da amplitude do deslocamento do centro de pressão ântero-posterior (COPap) e médio-lateral (COPml) durante os seis testes de organização sensorial (TOS). Santa Maria, RS, 2009.

\begin{tabular}{|c|c|c|c|}
\hline Condição (TOS) & $\mathrm{n}$ & COPap (cm) & $\mathrm{COPml}(\mathrm{cm})$ \\
\hline 1 & 38 & $1,42 \pm 0,50^{\mathrm{a}}$ & $0,78 \pm 0,43^{\mathrm{A}}$ \\
\hline 2 & 39 & $1,95 \pm 0,84^{\mathrm{ab}}$ & $1,08 \pm 0,65^{\mathrm{A}}$ \\
\hline 3 & 39 & $2,50 \pm 1,15^{\mathrm{b}}$ & $0,97 \pm 0,56^{\mathrm{A}}$ \\
\hline 4 & 39 & $2,56 \pm 1,14^{b}$ & $1,64 \pm 1,17^{\mathrm{B}}$ \\
\hline 5 & 39 & $4,11 \pm 1,62^{\mathrm{c}}$ & $2,25 \pm 1,06^{\mathrm{BC}}$ \\
\hline 6 & 39 & $5,70 \pm 2,28^{c}$ & $2,72 \pm 1,21^{\mathrm{C}}$ \\
\hline
\end{tabular}

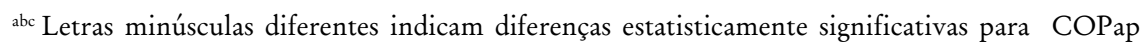

${ }^{\mathrm{ABC}}$ Letras maiúsculas diferentes indicam diferenças estatisticamente significativas para COPml

Para a realização das comparações, houve perda de uma tentativa de um indivíduo no TOS 1 , a qual foi retirada da análise descritiva e das comparações.

De acordo com os resultados apresentados na tabela 2, pode-se observar que para a direção ântero-posterior (COPap) não foram encontradas diferenças estatisticamente significativas, considerando-se o TOS 2 comparado ao TOS 1 , 3 e ao 4 e; o TOS 5 comparado ao TOS 6.

Com relação à amplitude do deslocamento médio-lateral (COPml), não foram encontradas diferenças estatisticamente significativas comparando-se o TOS 1 com o TOS 2 e 3; o TOS 2 comparado ao TOS 3; e o TOS 5 com o TOS 4 e TOS 6.

\section{DISCUSSÃO}

O presente estudo objetivou avaliar o equilíbrio corporal de mulheres idosas durante a manipulação dos sistemas sensoriais. Uma das limitações do estudo que podem ser citadas é com relação à amostra não probabilística utilizada. Assim, sugere-se que em futuros estudos um número maior de indivíduos seja avaliado, visando também à possibilidade de alcance de valores considerados como tendo uma distribuição normal.

A informação sensorial pode alterar o equilíbrio corporal em indivíduos idosos, uma vez que a manipulação dos sistemas envolvidos no controle postural proporciona mudança no equilíbrio. ${ }^{14}$ Essa afirmação associa-se aos 
resultados encontrados, visto que houve tendência em diferença de valores com a comparação das diferentes condições sensoriais.

De forma geral, ao analisar os resultados se pode perceber que o aumento do número de sistemas sensoriais manipulados faz com que haja maiores variações do COP. Segundo Schöner ${ }^{15}$ para que o controle postural atue com sucesso, é necessário que além de uma integração sensorial, ocorra uma relação coerente e estável entre a própria informação sensorial e a ação motora executada. Durante o TOS 1 (sem manipulação sensorial), foram encontrados os menores valores do COP na direção ântero-posterior (COPap), quando comparados às demais condições (TOS 2, 3, 4, 5 e 6), o que indica maior facilidade de manutenção do equilíbrio quando não há manipulação sensorial. Já no deslocamento médio-lateral no TOS 1 , os valores do COP das mulheres idosas não foram significativamente melhores apenas para os TOS 2 e 3 (com ausência da visão e com manipulação do cenário visual, respectivamente). Na comparação do TOS 1 com o 3 , os resultados podem ter relação com o fato de que em ambas as situações os indivíduos foram desafiados a manterem o equilíbrio corporal utilizando todas as vias aferentes para controle da estabilidade, ${ }^{15}$ o que aproximaria a dificuldade exigida para a tarefa. No caso do TOS 3, o que ocorre é que o cenário visual está em movimento e pode proporcionar conflito do sistema vestibular em identificar o real posicionamento do corpo no espaço. ${ }^{9}$

Além disso, outra explicação para a igualdade de valores na direção médio-lateral entre o TOS 1 e o TOS 3, é o fato que no TOS 3 há movimentação da cabine na direção ânteroposterior e isso pode ter gerado alteração no comportamento da oscilação nessa mesma direção. Ou seja, um aumento ântero-posterior e uma manutenção ou aumento não significativo da oscilação na direção médio-lateral dos indivíduos.

Esses resultados já foram visualizados na literatura consultada, sendo evidenciados no estudo de Ruwer ${ }^{16}$ quando diferentes faixas etárias foram avaliadas. No entanto, o estudo de Ruwer ${ }^{16}$ permite apenas a identificação dos valores relacionados à direção ântero-posterior, tornandose uma das limitações do uso da posturografia sem a utilização da plataforma de força.

Para Freitas Júnior \& Barela, ${ }^{9}$ as movimentações no cenário visual, durante uma coleta de equilíbrio, causam alterações no fluxo óptico, levando o indivíduo a produzir oscilações corporais relacionadas ao movimento do estímulo visual, para se manter o quadro de referência estabelecido que implique manutenção da estabilidade do cenário visual projetado na retina.

Wade et al. ${ }^{3}$ confirmam as indicações de Freitas Júnior \& Barela, ${ }^{9}$ ao relatarem que os idosos oscilam mais quando submetidos aos movimentos do cenário visual, devido, entre outros fatores, à diminuição da capacidade do sistema somatossensorial e vestibular em captar movimentos corporais, o que leva ao aumento das oscilações corporais.

Peterka et al. ${ }^{17}$ relataram que indivíduos saudáveis em ambiente iluminado e com uma base de suporte firme dependem $70 \%$ da informação somatossensorial, $10 \%$ visual e $20 \%$ vestibular, para a orientação postural. Porém, ao colocá-los em superfície instável, o peso da informação sensorial vestibular e visual aumenta, e diminui a dependência sobre a superfície da entrada somatossensorial. Neste caso, pode-se entender os motivos das maiores oscilações no TOS 5 e 6, já que mais de um sistema sensorial é acometido e/ou suprimido em um mesmo teste.

Essas afirmações refletem os achados de Wolfson et al., ${ }^{18}$ que observaram em cinco dos seis testes de organização sensorial realizados, uma significativa e maior oscilação em idosos saudáveis. Além disso, os autores encontraram grande proporção de quedas e maiores diferenças no TOS 5 e 6, quando comparados aos outros testes. Assim como no presente estudo, os autores verificaram diminuição do equilíbrio nos indivíduos idosos, quando o sistema visual e proprioceptivo foram suprimidos e/ou manipulados.

Jamet et al. ${ }^{19}$ realizaram um estudo com 40 indivíduos idosos e avaliaram o aumento da 
dependência visual devido à perturbação no controle do equilíbrio, durante a execução de dois tipos de tarefas cognitivas, uma tarefa visuo-verbal e uma tarefa mental. Os resultados mostraram aumento significativo nos valores de equilíbrio durante a condição de olhos fechados, mostrando maior desequilíbrio corporal. Assim, pode-se perceber a dependência da informação visual para idosas ativas, e esta se reflete nos valores encontrados em situação semelhante (TOS 5).

Desta forma, este estudo corrobora com os resultados encontrados, identificando diferenças entre os TOS $1,2,3$, e 4 na direção ânteroposterior e TOS 1, 2 e 3 na direção médio-lateral, quando comparados ao TOS 5 , mostrando aumento da oscilação corporal em ambas as direções (COPap e COPml) para condição de olhos fechados.

Mann et al. ${ }^{20}$ também encontraram resultados semelhantes, ao investigarem o equilíbrio corporal de 20 idosos praticantes de hidroginástica e 15 indivíduos adultos sedentários. Os autores, ao manipularem a informação visual, encontraram diferenças estatisticamente significativas para ambos os grupos, destacando assim a importância da informação visual para idosos.

Nas comparações realizadas entre todas as posições e aquelas em que se utilizou conflito proprioceptivo (TOS 4, TOS 5 e TOS 6), observou-se que para o COPap, das 12

\section{REFERÊNCIAS}

1. Fleck MPA, Chachamovich E, Trentini CM. Development and validation of the Portuguese version of the WHOQOL-OLD module. Rev Saúde Publica 2003 oct; 37(6): 793-799.

2. Ruwer SL, Rossi AG, Simon LF. Equilíbrio no idoso. Rev Bras Otorrinolaringologia 2005 mai/ jun; 71(3): 298-303.

3. Wade $\mathrm{M}$, et al. Optical flow, spatial orientation, and the control of posture in the elderly. Psychological Science 1995; 50(1): 51-58.

4. Sundermier $\mathrm{L}$, et al. Postural sensitivity to visual flow in aging adults with and without balance problems. J. Gerontol 1996 mar; 51(2): 45-53. comparações 10 foram significativas e, para o COPml 10, foram significativas, sendo que em ambos a média do deslocamento foi maior para essas três posições em todas as comparações. Logo, em superfícies instáveis, há alteração da sensação proprioceptiva, diminuindo neste caso a estabilidade das idosas.

Nesse contexto, Gauchard et al..$^{21}$ explicam que, estando o indivíduo em condições normais, a propriocepção e a informação sensorial da superfície cutânea plantar são fontes importantes de sistemas sensoriais para manutenção do controle postural. Além disso, para Perrin et al..$^{22}$, o sistema proprioceptivo é o principal sistema sensorial envolvido na manutenção do controle de equilíbrio corporal.

\section{CONSIDERAÇÕES FINAIS}

De acordo com os resultados encontrados no presente estudo, observa-se que a informação sensorial pode alterar a manutenção o equilíbrio corporal em idosas fisicamente ativas. De forma geral, quanto mais sistemas sensoriais forem manipulados, pior será o equilíbrio corporal. $\mathrm{O}$ TOS 6, no qual há manipulação do cenário visual e alteração proprioceptiva por meio da almofada, demonstrou ser a condição de maiores instabilidades na posição ortostática, tanto na direção ântero-posterior quanto na direção médio-lateral.
5. Bittar RSM, et al. Síndrome do desequilíbrio no idoso. Pró-Fono 2002 jan/abr; 14 (1): 11928.

6. Lord SR, Sherrington C, Menz HB. Falls in older people: risk factors and strategies for prevention. Cambridge: Cambridge University Press; 2001.

7. Kron M, et al. Risk indicators for falls in institutionalized frail elderly. Am J Epidemiol 2003 apr; 158(7): 645-653.

8. Tinetti ME, et al. Yale Ficsit: risk factor abatement strategy for fall prevention. J Am Geriatrics Society 1993 mar; 41(3): 315-320. 
9. Freitas JP, Barela JA. Alterações no funcionamento do sistema de controle postural de idosos: uso da informação visual. Rev Portuguesa de Ciência do Desporto 2006; 6(1): 94-105.

10. Mochizuki L, Amadio AB. As informações sensoriais para o controle postural. Fisioterapia em movimento $2006 \mathrm{abr}$./jun; 19(2): 11-18.

11. Carvalho RL, Almeida GL. Aspectos sensoriais e cognitivos do controle postural. Rev Neurociência 2009; 17(2): 156-60.

12. Benedetti TRB, Mazo GZ, Barros MV. Aplicação do Questionário internacional de atividade física para avaliação do nível de atividades físicas de mulheres idosas: validade concorrente e reprodutibilidade teste/reteste. Rev Bras Ciênci Movimento 2004; 12(1): 25-33.

13. Castagno LA. A new method for sensory organization tests: the foam-laser dynamic posturography. Rev Brás Otorrinolaringologia 1994; 60(4):287-296.

14. Jeka JJ, Oie K, Kiemel KS. Multisensory information for human postural control: Integrating touch and vision. Experimental Brain Research 2000 set; 134(1): 107-125.
15. Schöner G. Dynamic theory of actionperception patterns: the moving room paradigm. Biological Cybernetics 1991 apr; 64(6): 455-462.

16. Ruwer SL. Estudo da posturografia dinâmica : "Foam Laser" em indivíduos normais com idade entre 14 e 60 anos. [Dissertação de Mestrado] Santa Maria: Universidade Federal de Santa Maria, Distúrbios da Comunicação Humana; 2006.

17. Peterka RJ. Sensorimotor integration in human postural control. J of Neurophysiology 2002 may; 88(3): 1097-1118.

18. Wolfson L, et al. A dynamic posturography study of balance in healthy elderly. Neurology 1992 nov; 42(11): 2069-2075.

19. Jamet $\mathrm{M}$, et al. Higher visual dependency increases balance control perturbation during cognitive task fulfilment in elderly people. Neuroscience Letters 2004 feb; 359(1-2): 61-64.

20. Mann L, et al. Investigação do equilíbrio corporal em idosos. Rev Bras Geriatri Gerontol 2008 mai/ago; 11(2): 155-165.

21. Gauchard GC, et al. Physical activity improves gaze and posture control in the elderly. Neuroscience Research 2003; 45(4): 409-417.

22. Perrin PP, et al. Effects of physical and sporting activities on balance control in elderly people. Briti J Sports Med 1999; 33(2): 121-126. 